\title{
Accurate atomic parameters for near-infrared spectral lines
}

\author{
J. M. Borrero ${ }^{1}$, L. R. Bellot Rubio ${ }^{2}$, P. S. Barklem ${ }^{3}$, and J. C. del Toro Iniesta ${ }^{4}$ \\ 1 Max-Planck Institut für Aeronomie, 37191 Katlenburg-Lindau, Germany \\ 2 Kiepenheuer-Institut für Sonnenphysik, Schöneckstr. 6, 79104 Freiburg, Germany \\ 3 Uppsala Astronomical Observatory, Box 515, 751-20 Uppsala, Sweden \\ ${ }^{4}$ Instituto de Astrofísica de Andalucía, CSIC, Apdo. de Correos 3004, 18080 Granada, Spain
}

Received 31 March 2003 / Accepted 10 April 2003

\begin{abstract}
A realistic two-component model of the quiet solar photosphere is used to fit the intensity spectrum of the Sun in the wavelength range $0.98-1.57 \mu \mathrm{m}$. Our approach differs from earlier attempts in many respects: proper account of convective inhomogeneities is made, accurate collisional broadening parameters from quantum mechanical computations are used, and the effects of possible blends in the local continuum are corrected empirically. This allows us to derive oscillator strengths and central wavelengths for virtually any unblended line of the solar spectrum. The accuracy of the inferred atomic parameters, about 0.06 dex for oscillator strengths and $5 \mathrm{~m} \AA$ at $1 \mu \mathrm{m}$ for central wavelengths, is similar to that of the best laboratory measurements. We apply our method to 83 near-infrared lines belonging to 6 different atomic species. The availability of accurate oscillator strengths and central wavelengths for lines of different species is essential for the interpretation of high resolution spectroscopic observations. The method is especially useful in the infrared, a wavelength domain where laboratory measurements are scarce.
\end{abstract}

Key words. atomic data - line: profiles - Sun: abundances - stars: abundances

\section{Introduction}

Light emitted by atoms is the primary source of information in all branches of astrophysics. The intensity and polarization spectra emerging from celestial objects encode information on the physical conditions of the medium where the spectral lines are formed. Usually, this information is extracted by adopting a suitable model, computing synthetic line profiles for the physical conditions of the model, and comparing them with the observations. Spectroscopy is ultimately tied to our knowledge of atomic physics in many ways; not only as far as the interaction of light with atoms and molecules is concerned, but also because accurate atomic data are required for a correct interpretation of the observations.

Many groups have worked intensively in the last decades to provide the scientific community with accurate atomic parameters. The methods employed range from purely theoretical and semiempirical calculations (e.g., Seaton et al. 1994; Kurucz 1995) to entirely experimental techniques. Very precise laboratory measurements have been carried out in Oxford (Blackwell et al. 1986, and references therein) and Hannover (Bard et al. 1991; Bard \& Kock 1994). Laboratory measurements have also been performed by other groups (e.g., May et al. 1974; O’Brian et al. 1991; Nave et al. 1994). A different approach based on the fitting of the solar spectrum has been explored by, among

Send offprint requests to: J. M. Borrero,

e-mail: borrero@linmpi.mpg.de others, Gurtovenko \& Kostik (1981, 1982), Thévenin (1989, 1990), and Bellot Rubio et al. (1999). Most of these efforts have concentrated on the ultraviolet and visible regions of the electromagnetic spectrum (from $1400 \AA$ to $8000 \AA$ ) and only for a number of atoms of relative importance in astrophysics (e.g., $\mathrm{Fe}, \mathrm{Si}, \mathrm{Ti}, \mathrm{Cr}$ ). Unfortunately, the absolute scales and accuracies of the available oscillator strengths and central wavelengths are rather inhomogeneous, and many atomic species still need to be measured (see Kurucz 2003 for a review).

The situation is worse in the infrared, where laboratory measurements are scarce and theoretical calculations give order-of-magnitude estimates only. This is unfortunate in view of the increasing interest of the astrophysical community in this part of the spectrum. A number of state-of-the-art infrared instruments have been developed during the last years, including the Tenerife Infrared Polarimeter (TIP, Martínez Pillet et al. 1999) at the German Vacuum Tower Telescope of Teide Observatory and the Cryogenic high-Resolution Infrared Echelle Spectrograph (CRIRES, Wiedemann et al. 2000) at the Very Large Telescope of Paranal Observatory. For the determination of magnetic fields via spectropolarimetry, infrared lines are preferred because of their large Zeeman splittings. In abundance studies of cool stars, the infrared is advantageous because it is cleaner than the visible part of the spectrum.

Clearly, many investigations would benefit from accurate atomic parameters in the near infrared. However, until laboratory measurements become available, the only way 
to determine such parameters with reasonable degree of accuracy is to use the Sun as a laboratory. This work is part of a continuing effort toward that goal. Here we present a list of accurate central wavelengths and oscillator strengths for 83 unblended lines pertaining to 6 different atomic species in the wavelength range $0.98-1.57 \mu \mathrm{m}$. The atomic parameters have been determined by fitting the intensity spectrum of the Sun. The main difference between our work and earlier investigations is that we use a realistic model of the solar photosphere (Borrero \& Bellot Rubio 2002; hereafter BBR) together with a more appropriate treatment of the broadening of spectral lines by collisions with neutral hydrogen (Anstee \& O'Mara 1995; Barklem \& O'Mara 1997; Barklem et al. 1998; hereafter ABO). Another improvement is that we fit the intensity profiles of the spectral lines, not their equivalent widths. In Sect. 2 we briefly discuss the various methods employed so far for the determination of atomic parameters, emphasizing their advantages and disadvantages, and quantifying the typical uncertainties associated with them. A detailed description of our method is given in Sect. 3. Section 4 presents the oscillator strengths and central wavelengths of 83 infrared lines, many of which have interesting diagnostic potentials for astrophysical applications. In Sect. 5 we evaluate the accuracy of the inferred atomic parameters. Finally, Sect. 6 summarizes the conclusions of this work.

\section{Determination of atomic parameters}

Broadly speaking, there are three methods for obtaining transition probabilities and central wavelengths, namely direct laboratory measurements, theoretical determinations based on atomic structure computations, and measurements of spectral lines in the solar spectrum. In what follows we briefly describe these methods and point out some of their strengths and limitations.

\subsection{Transition probabilities}

Laboratory measurements. The determination of relative oscillator strengths in the laboratory is based on measurements of the intensity of spectral lines either in absorption (e.g., Blackwell \& Collins 1972) or emission (e.g., May et al. 1974). The main source of error in these determinations is the imprecise knowledge of the temperature of the light source, which is needed to compute the number of absorbing or emitting atoms under the assumption of thermodynamic equilibrium. Other problems are discussed by Blackwell (1990). The relative oscillator strengths obtained from such intensity measurements are referred to an absolute scale using precise oscillator strengths estimated by other means for some of the observed lines.

Another possibility is to combine the normalized relative intensities of lines arising from the same upper level (the so-called branching fractions) with absolute measurements of the level lifetime. Time-resolved, laser-induced fluorescence (e.g., O'Brian et al. 1991) is a popular technique for determining radiative lifetimes with accuracies of about $5 \%$. Highprecision lasers are used to excite the atomic level of interest. The spontaneous radiative decay is observed over time, and from the decay curve an exponential lifetime is computed.
The combination of branching fractions and radiative lifetimes is generally regarded as the most reliable method for obtaining accurate oscillator strengths (O'Brian et al. 1991; Bard et al. 1991; Bard \& Kock 1994). The absolute oscillator strengths determined in this way are independent of any assumption concerning the thermodynamic state of the source because lines having a common upper level are used. However, it is necessary that the lines be emitted from an optical thin layer in order to avoid self-absorption. With this technique, $g f$-values can be measured with an uncertainty of 5-10\%.

Highly excited atomic levels are difficult to populate in the laboratory. For this reason, the various techniques mentioned so far are more appropriate for characterizing atomic transitions occurring in the UV and visible part of the spectrum. Among all published transition probabilities, the values given by the Oxford and Hannover groups are considered to be the most accurate, with a remarkable internal consistency. A comparison of the oscillator strengths of 23 spectral lines measured by the two groups can be found in Bard et al. (1991). The deviations between two sets of oscillator strengths can be quantified in terms of the mean $\Delta$ and the standard deviation $\sigma$ of the differences between individual values. We note that $\sigma=0.1 \mathrm{dex}$ is equivalent to an rms difference of about $25 \%$ in the individual $g f$-values. Bard et al. (1991) found that Oxford and Hannover oscillator strengths for neutral iron lines are essentially the same ${ }^{1}$, with $\Delta=0.03$ and $\sigma=0.06$ dex. Such an rms difference is consistent with the uncertainties of the measurements. Typical uncertainties are between 0.03 and $0.05 \mathrm{dex}$ ( $7 \%$ and $12 \%$, respectively), although individual $g f$-values may be uncertain by up to 0.11 dex.

A comparison between the laboratory measurements of Oxford and Hannover with those of O'Brian et al. (1991) and May et al. (1974) for the same set of Fe I lines is presented in Fig. 1. The rms difference $\sigma$ amounts to 0.15 dex in the case of O'Brian et al. (1991) and 0.12 dex in the case of May et al. (1974). The absolute scales agree rather well, with $\Delta=0.00$ and $\Delta=-0.02$ dex, respectively. The larger scatter of O'Brian et al. data might be due to the fact that some of their transitions probabilities were obtained from interpolated level populations. The small offset in the absolute scale of May et al. (1974) could be due to problems with the calibration of their emission measurements. In view of these comparisons, it seems appropriate to think of the oscillator strengths measured at Oxford and Hannover (Blackwell et al. 1982, 1986; Bard et al. 1991; Bard \& Kock 1994; and references therein) as reference values. Experimental work aimed at completing the database of oscillator strengths for elements of the iron group is being carried out within the framework of the FERRUM project (Johansson 2002), mainly in the UV.

Theoretical calculations. Oscillator strengths have been computed for essentially all lines of astrophysical interest within the Opacity Project (Seaton et al. 1994). These values are based on

\footnotetext{
${ }^{1}$ Elements such as $\mathrm{Cr}$ and $\mathrm{Ti}$ have also been studied exhaustively, but most of the efforts have been devoted to iron because of its astrophysical importance. As a result, the more precise oscillator strengths available are those of neutral iron.
} 


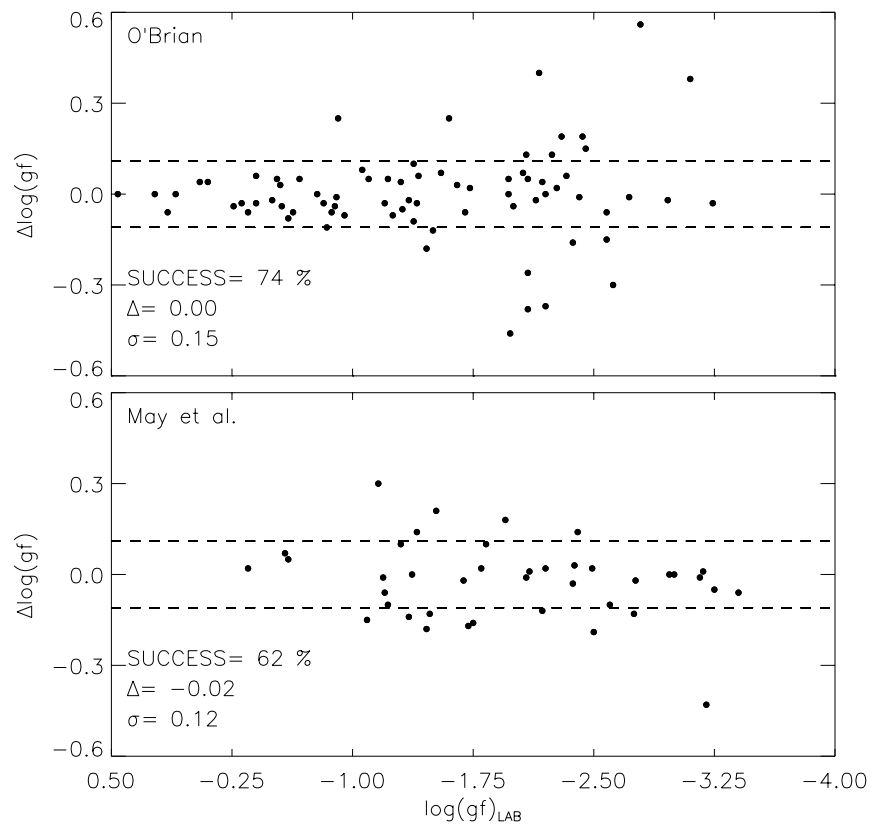

Fig. 1. Comparison of oscillator strengths determined by O'Brian et al. (1991, top) and May et al. (1974, bottom) with laboratory measurements from Oxford and Hannover for a list of common Fe I lines in the UV and visible. The rms deviations with respect to the reference values are $\sigma=0.15 \mathrm{dex}$ and $\sigma=0.12 \mathrm{dex}$, respectively. The horizontal dashed lines represent deviations of \pm 0.1 dex. This is the $25 \%$ error adopted in earlier comparisons of laboratory measurements (e.g., Bard et al. 1991). The percentage of oscillator strengths differing from the reference values by less than 0.1 dex is indicated as success.

ab initio calculations of atomic wavefunctions assuming pure LS coupling. The computed transition probabilities may be in error by a factor of two or more. Significantly higher precision can be achieved if experimental energy levels are used to constrain the atomic wavefunctions. The basic idea is to fit the eigenvalues of a parametrized model Hamiltonian to the observed energies. The Hamiltonian resulting from the fit is diagonalized in order to find its eigenvectors. The eigenvectors are used to transform the pure LS transition matrix into the actual intermediate coupling scheme. Finally, the elements of the transformed matrix give the desired $g f$-values. Semiempirical determinations of this kind have been carried out intensively by, e.g., Kurucz \& Peytremann (1975). Use of Kurucz values is widespread in the literature due to the huge number of atomic transitions that have been characterized in this way, not only in the UV and visible parts of the spectrum, but also in the infrared, for virtually all atoms and all ionization stages. Unfortunately, these values can be in error by as much as 0.2-0.5 dex according to Blackwell et al. $(1976,1979)$. Assessing the accuracy of semiempirical oscillator strengths is beyond the scope of this work. However, it is clear that a basic limitation is the precision with which the experimental energies are known. A refinement of the method described above involves the use of orthogonal operators in order to stabilize the fits to the observed energies (see Uylings \& Raassen 1997, and references therein). This makes it possible to consider higher order effects in the model, which results in significant improvements in the accuracy of the eigenvectors, and hence in the final oscillator strengths. The orthogonal operator method has been applied to a few lines only.

Solar measurements. Oscillator strengths from the solar spectrum have been an alternative to laboratory measurements whenever large numbers of lines were needed. The extremely high vacuum conditions $\left(\rho=10^{-7}-10^{-8} \mathrm{~g} \mathrm{~cm}^{-3}\right.$ ) and high temperatures $\left(10^{3}-10^{4} \mathrm{~K}\right)$ of the solar atmosphere provide conditions for many atomic and molecular transitions to appear clearly in the spectrum of the Sun. Solar oscillator strengths have been obtained sucessfully by, among others, Gurtovenko \& Kostik $(1981,1982)$ and Thévenin $(1989,1990)$. The underlying method is to use a suitable model of the solar photosphere and a given set of elemental abundances to solve the radiative transfer equation and generate synthetic spectral lines which are compared with the spectrum emerging from the quiet Sun. If the model atmosphere is realistic enough, any discrepancy between synthetic and observed spectral lines can be ascribed to incorrect oscillator strengths that are modified until the best fit is achieved. The application of this simple idea turns out to be very difficult, mainly because of our inability to model the solar photosphere with sufficient realism. Among the various simplifications adopted, the most important are perhaps the use of one-component models of the solar photosphere (e.g., Gingerich et al. 1971; Holweger \& Müller 1974; Gustafsson et al. 1975), the approximate treatment of the broadening of spectral lines by atomic collisions based on Unsöld (1955) theory, and the assumption of local thermodynamical equilibrium. None of these simplifications is strictly valid. As a consequence, several free parameters had to be invoked: damping enhacement factors, micro- and macroturbulent velocities, etc. It is perhaps for this reason that solar oscillator strengths have never been given much credibility outside the solar physics community. In the present paper we argue that solar oscillator strengths may be as accurate as laboratory measurements. This is already demonstrated in Fig. 2, where the values determined by Gurtovenko \& Kostik and Thévenin are compared with the laboratory measurements from Oxford and Hannover. The rms difference between the solar and experimental values is on the order of $\sigma=0.15$ dex, i.e., similar to the uncertainties of the laboratory measurements presented in Fig. 1. This result is quite remarkable in view of the simplifications of the method. In Sect. 5 we demonstrate that removal of some of the assumptions leads to significant improvements in the accuracy of the oscillator strengths derived from the solar spectrum.

\subsection{Central wavelengths}

Laboratory measurements. Central wavelengths are measured almost exclusively from emission spectra generated by low pressure sources such as hollow cathode lamps. The absolute wavelength scale of the observations is set by reference lines whose wavelengths are known precisely by, e.g., interferometric techniques. It is important to use high-dispersion spectra in order to determine the exact position of the lines. Although grating spectrographs are appropriate for this purpose, the highest resolving power is provided by Fourier 




Fig. 2. Comparison of oscillator strengths determined by Gurtovenko \& Kostik (1981, 1982; top) and Thévenin (1989, 1990; bottom) with laboratory measurements from Oxford and Hannover for $80 \mathrm{Fe} I$ lines in the visible part of the spectrum. In both cases, less than $50 \%$ of the lines are reproduced within the uncertainties of laboratory measurements (horizontal dashed lines at $\pm 0.1 \mathrm{dex}$ ). The rms differences with respect to the laboratory values are $\sigma=0.14$ dex for Gurtovenko \& Kostik data, and $\sigma=0.15$ dex for Thévenin data.

Transform spectrometers (see Litzén 1995 for a review). Learner \& Thorne (1988) give a detailed account of possible wavelength shifts in this kind of measurements. The most important systematic error is the absolute wavelength calibration of the spectra. Photon noise is the main source of random errors. Weak lines are difficult to measure, implying that their wavelengths are known with less accuracy than those of strong lines. The element for which the most accurate laboratory measurements exist is neutral iron. Nave et al. (1994) give precise wavelengths for $9501 \mathrm{Fe}$ I lines observed in high resolution spectra covering the interval $1700 \AA-5 \mu \mathrm{m}$. The uncertainties of these measurements are quoted to be on the order of $0.005 \mathrm{~cm}^{-1}(\sim 1.25 \mathrm{~m} \AA$ at $5000 \AA$ and $\sim 5 \mathrm{~m} \AA$ at $1 \mu \mathrm{m})$ for the strongest lines. Laboratory wavelengths for other species and ionization stages may be much more uncertain. In the case of Fe II, for example, uncertainties of up to $20 \mathrm{~m} \AA$ have been reported (Johansson 1978). Over the last years, high resolution Fourier transform spectra of neutral and ionized $\mathrm{Fe}, \mathrm{Cr}$, $\mathrm{V}, \mathrm{Co}$, and other elements of the iron group have been obtained at Imperial College and Lund University (see Johansson 1995; Pickering et al. 2002). The analysis of these data has improved the accuracy of existing laboratory wavelengths by as much as one order of magnitude. So far, the efforts have concentrated on the UV part of the spectrum.

Theoretical calculations. Ritz wavelengths can be predicted from calculated energy levels, as done by Kurucz \& Peytremann (1975). However, these values may be wrong by



Fig. 3. Comparison of central wavelengths determined from the solar spectrum using the two-component model of BBR and laboratory wavelengths of Nave et al. (1994) for a set of 950 lines of neutral iron in the visible range. The rms differences between solar and laboratory values are given in $\mathrm{m} \AA$ above the $x$-axis for a number of wavelength intervals.

several angstroms because of inaccurate eigenvectors. In general, only experimental energy levels can be used with confidence for the determination of wavelengths.

Solar measurements. The solar spectrum can be used to infer very accurate central wavelengths for virtually any atomic transition. However, this is a delicate task. Spectral lines in the solar spectrum are shifted from their laboratory positions not only because of the gravitational field of the Sun (around $636 \mathrm{~m} \mathrm{~s}^{-1}$ ), but also because of convective motions. Such motions shift the spectral lines toward shorter wavelengths in amounts that depend on the height of formation of the lines. These convective blueshifts have to be removed in order to determine accurate central wavelengths from the solar spectrum. One-component models of the photosphere neglect convective inhomogeneities, so they are of little use for this purpose. More complex twocomponent models such as that of BBR are able to describe convective motions to a very reasonable degree of accuracy. The first applications of this kind of models (Bellot Rubio et al. 1999; Frutiger et al. 2000) revealed that the Fe II absolute wavelength scale of Johansson (1978) is off by about $-6 \mathrm{m \AA}$ with respect to that defined by Nave et al. (1994) for Fe I. Figure 3 allows us to estimate the accuracy of the central wavelengths determined from the solar spectrum. In that figure, the solar wavelengths of $950 \mathrm{Fe}$ I lines measured by BBR are compared with the laboratory wavelengths of Nave et al. (1994). The maximum rms difference between them is $\sigma \sim 1.6 \mathrm{m \AA}$. This difference comes from uncertainities in the solar and laboratory wavelengths according to $\sigma^{2}=\sigma_{\text {solar }}^{2}+\sigma_{\text {Nave }}^{2}$. Inserting the numbers given by Nave et al. (1994), we find that the typical uncertainty of the wavelengths estimated by BBR from the solar spectrum is on the order of $1 \mathrm{~m} \AA$ in the visible. The high accuracy of solar determinations has important practical consequences. For example, the term system of a given atom or ion can be improved and extended if very precise wavelengths are 
measured for a sufficiently large number of lines (see, e.g., the recent term analysis of Co II by Pickering et al. 1998).

\section{Description of the method}

The determination of atomic parameters from the solar spectrum involves a comparison between synthetic and observed spectral lines. The synthetic lines are computed integrating the radiative transfer equation in a prescribed model of the solar photosphere. Any difference between the synthetic and observed profiles is ascribed to incorrect transition probabilities and central wavelengths, which can be modified iteratively until the best fit is reached. In this section we concentrate on radiative transfer issues, and describe the model atmosphere, the synthesis/inversion code, the collisional broadening theory, and the treatment of blends in the local continuum that we use for the determination of atomic parameters. In earlier work, the comparison between observed and synthetic profiles was based on equivalent widths and/or line core intensities given the inability of one-component models to reproduce the shape of the spectral lines. This is no longer a limitation, so our analysis is based on profile-fitting techniques in order to increase the reliability of the inferred atomic parameters.

\subsection{Model atmosphere}

The quiet solar photosphere is highly inhomogeneous. Convective cells penetrating from the convection zone cause a rich variety of intensity and velocity patterns. Inhomogeneities not only occur in the horizontal direction, but also in the vertical direction. All these structures leave their imprints on the emergent spectrum. One-component models of the photosphere neglect this structuring, and therefore they are unable to explain many features of the solar spectrum. Prominent examples include the convective blueshifts and $C$-shaped bisectors of photospheric lines. Other features such as the convective broadening of the solar lines are modeled using ad hoc parameters like, for example, microturbulent and macroturbulent velocities. Given the empirical nature of these parameters, it is necessary to vary them for each line so as to provide the best fit to the observations. Determinations of chemical abundances and atomic parameters based on one-component models are known to be affected by systematic errors (Bellot Rubio \& Borrero 2002; Steffen \& Holweger 2002; see also the clear trend with line strength in our Fig. 2). The granular inhomogeneities of the solar photosphere can be modeled in a realistic way by means of 3D, fully compressible, hydrodynamical simulations at high Reynolds numbers including non-grey radiative transfer, NLTE effects, and partial ionization of the different species. Current numerical simulations have reached a high degree of sophistication. They are able to reproduce, among other observables, the granular patterns, the spectral line shapes and bisectors, and the frequencies of the p-mode oscillations of the photosphere (Asplund et al. 2000a,b; Stein \& Nordlund 1998). However, these simulations are not very well suited to the determination of atomic parameters because of the computational effort required to produce spatially and temporally averaged synthetic profiles out of the high spatial and temporal resolution snapshots. For a systematic analysis of the solar spectrum, a simplified but still realistic description of the solar surface is more appropriate.

Recently, we have presented a two-component model of the solar photosphere (BBR). This model provides a simple description of the solar granulation in terms of two atmospheric components representing spatially and temporally averaged granules and intergranules (hot plasma upflows and cool downflows, respectively). The two-component model was obtained from a LTE inversion of 22 neutral iron lines for which very accurate atomic parameters (oscillator strengths, central wavelengths, and collisional parameters) were known. In addition, the observations were taken from the same spectral atlas we employ here (see Sect. 4.2). Thus, the model is representative of the very same physical conditions under which the lines to be analyzed were formed. Despite its simplicity, the two-component model is able to reproduce the line shifts and equivalent widths of about 800 visible lines of the solar spectrum. It is also capable of matching the center-to-limb variation of the continuum intensity with higher accuracy than any one-component model. Exploratory calculations have shown that it can be employed to infer oscillator strengths with an accuracy comparable with that of the best laboratory measurements (BBR). Bellot Rubio \& Borrero (2002) have used this model to determine the solar iron abundance. The abundances resulting from individual Fe I and Fe II lines do not depend on line strength, and are fully consistent with those estimated from more complex 3D numerical simulations (Asplund et al. 2000c).

Unlike one-component models, BBR's model is able to reproduce the asymmetrical shapes and broadening of the spectral lines induced by convective motions. This allows us to fit the full shape of the observed lines in order to increase the amount of information. Successful profile fits mean that equivalent widths, line core intensities, and bisectors are also reproduced. At this point we note that our model does not give a perfect description of the real Sun. Therefore, we still have to use macroturbulent and microturbulent velocities. However, there is a substantial difference between our treatment and those of authors using one-component models. Gurtovenko \& Kostik (1981, 1982), for example, considered macroturbulence and microturbulence as free parameters, that is, they adopted different values for weak and strong lines. We aim at a more consistent treatment, and keep the micro and macroturbulent velocities fixed at the values derived from the inversion of the $22 \mathrm{Fe}$ I lines (BBR). Changing these values arbitrarily would be inconsistent, as the two-component model would not be able to reproduce the spectral lines from which it was determined.

\subsection{Synthesis of spectral lines}

The spectral lines emerging from the two-component model are synthesized with SIR (Stokes Inversion based on Response functions; Ruiz Cobo \& del Toro Iniesta 1992). The synthesis module of SIR is based on an earlier code by Wittmann (1974) except for the integration of the radiative transfer 
equation, which is carried out using the Hermitian algorithm of Bellot Rubio et al. (1998). The basic assumptions are LTE, plane-parallel geometry, and hydrostatic equilibrium. The continuum absorption coefficient is evaluated for a given wavelength, temperature, and electron pressure taking into account contributions from $\mathrm{H}, \mathrm{He}, \mathrm{H}^{-}, \mathrm{He}^{-}, \mathrm{H}_{2}^{-}, \mathrm{H}_{2}^{+}, \mathrm{C}, \mathrm{Mg}$, and $\mathrm{Na}$, as well as Rayleigh scattering by $\mathrm{H}, \mathrm{H}_{2}$, and $\mathrm{He}$, and Thomson scattering by free electrons (for details, see Wittmann 1974). Electron pressures are put in hydrostatic equilibrium using the equation of state of an ideal gas with variable mean molecular weight to account for the partial ionization of the various atomic elements. Gas pressures are computed from temperatures and electron pressures on the assumption of LTE and chemical equilibrium.

In this work, several optimizations of SIR have been carried out. To account for the broadening of the spectral lines by collisions with neutral atoms, quantum mechanical parameters have been implemented in substitution of the classical Unsöld (1995) formula (see Sect. 3.3 for details). Radiative damping constants measured in the laboratory are used whenever possible, otherwise a damped harmonic oscillator is assumed. Stark broadening is neglected.

\subsection{Collisional broadening}

Collisions with neutral hydrogen atoms is an important mechanism for broadening lines in the solar amosphere, particularly in strong lines. Although charged particles such as electrons and ions interact more strongly and broaden lines more efficiently, in the atmospheres of cool stars like the Sun hydrogen atoms outnumber electrons by about four orders of magnitude and therefore dominate the collisional broadening of most photospheric metal lines. The classical Unsöld formula, which is based on the simple van der Waals potential $C / R^{6}$, is known from comparison to the observed broadening in the solar spectrum to typically underestimate the broadening by around a factor of two. Despite this, due to its relatively simple form and wide applicability compared with detailed calculations available for a few selected lines, it has remained in wide use in astrophysics, often with a correction or "enhancement" factor $E$ derived from the solar spectrum.

During the nineties, the ABO theory (Anstee \& O'Mara 1991; Anstee \& O'Mara 1995; Barklem \& O’Mara 1997; Barklem et al. 1998) was developed with the aim of describing collisional broadening as accurately as possible while also having the wide applicability desirable for astrophysical applications. The work provides a universal theory for the broadening of low-lying lines of neutral atoms. The most important features of the ABO formulation are the development of a method for computing the interatomic potential between the hydrogen atom and a generic neutral atom using perturbation theory with the unexpanded electrostatic interaction, and that the orientation of the pertubed atom ( $m$ state) is accounted for. This leads to interatomic potentials which are reliable at intermediate range (where the potential deviates from asymptotic van der Waals behavior) and long range, which are the interactions of importance in broadening by hydrogen. However, to keep the theory suitably general for wide application, some approximations must be made, such as the neglect of avoided crossings and exchange in the interaction potentials. Lack of reliable experimental data for this process makes it difficult to judge the accuracy of the ABO calculations with certainty. However, comparisons with the more detailed calculations available for some lines (e.g., Na D lines) and the solar spectrum indicate that the ABO theory is accurate to perhaps around $10 \%$ (e.g., Barklem \& O’Mara 2001).

The broadening data used in this work have been calculated from the ABO theory and are described by two parameters. The line broadening cross section $\sigma$ is given in atomic units for a collision speed of $v_{0}=10^{4} \mathrm{~m} \mathrm{~s}^{-1}$. The cross section for other velocities is calculated via the velocity parameter $\alpha$ which is derived from a fit to the velocity dependence of the cross sections computed for a range of velocities assuming that

$\sigma(v)=\sigma\left(v_{0}\right)\left(v / v_{0}\right)^{-\alpha}$.

The line half half-width for a given temperature and hydrogen number density is found from $\sigma$ and $\alpha$ using the expression

$\frac{w}{N}=\left(\frac{4}{\pi}\right)^{\alpha / 2} \Gamma\left(\frac{4-\alpha}{2}\right) v_{0} \sigma\left(v_{0}\right)\left(\frac{\bar{v}}{v_{0}}\right)^{1-\alpha}$,

where $\bar{v}=(8 k T / \pi \mu)^{1 / 2}$ and $\mu$ is the reduced mass of the two atoms.

For the majority of lines employed in this work broadening parameters were obtained by interpolating in the previously published tables of general broadening data for given effective principal quantum numbers (Anstee \& O’Mara 1995; Barklem \& O'Mara 1997; Barklem et al. 1998), taking particular care to correctly account for excited parent configurations in determining the effective principal quantum number (e.g., Barklem et al. 2000). For the Si I $12189 \AA$ A line the upper state was just outside the region of the tabulated data. While this is outside the region of strict validity of the theory due to the expected increased influence of exchange effects in the interaction, broadening data were calculated specifically, and should provide a reasonable estimate. Lines involving very excited states, where the theory is not applicable, were rejected.

\subsection{Inversion of observed lines}

The observed spectral lines are fitted by means of a leastsquares procedure using the inversion module of SIR. Each line is considered separately. The only parameters allowed to vary during the fit are the oscillator strength $g f$, the central wavelength $\lambda_{0}$, and a continuum correction factor (see Sect. 3.5). In order to speed up the inversion process, analytical response functions for oscillator strengths and central wavelengths have been implemented in SIR. As explained by Ruiz Cobo \& del Toro Iniesta (1994) and del Toro Iniesta (2003), response functions are the partial derivatives of the emergent intensity profile with respect to the free parameters of the inversion.

\subsection{Continuum correction}

Some regions of the solar spectrum are heavily populated by atomic lines. Molecules are also important in the infrared 

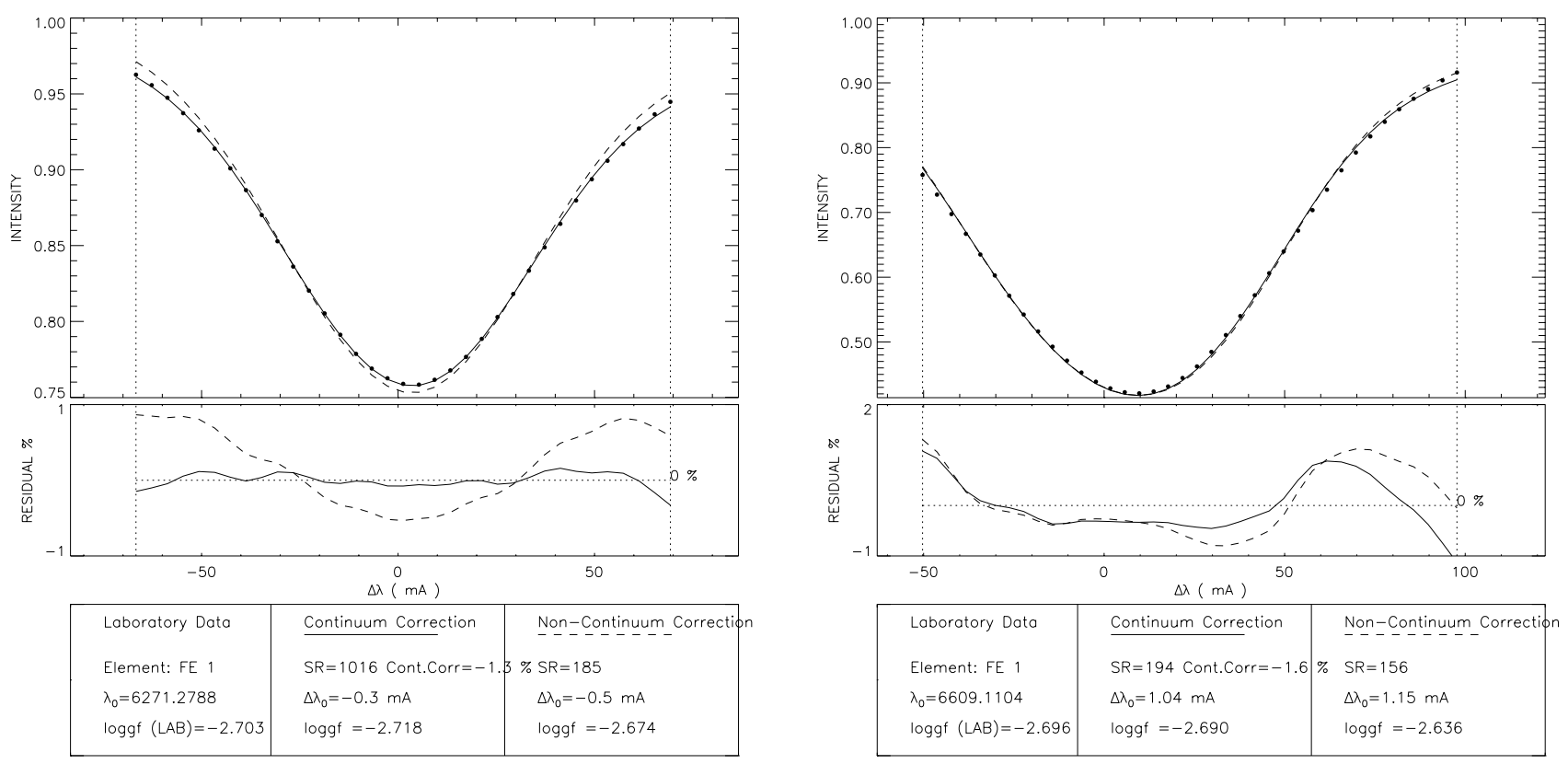

Fig. 4. Two examples of the determination of oscillator strengths $(\log g f)$ and central wavelengths $\left(\lambda_{0}\right)$ for neutral iron lines in the visible range. Left panels: Fe I 6271 A. Right panels: Fe I $6609 \AA$. The observations are represented by dots. The fits without continuum correction (dashed lines) are quite satisfactory. The residuals, shown in the lower panels, are about $1 \%$ at most, and the derived oscillator strengths differ from the laboratory values by $-0.03 \mathrm{dex}$ and $-0.06 \mathrm{dex}$, respectively. When the continuum correction is applied (solid lines), the fits and the inferred oscillator strengths improve. The differences between solar and laboratory oscillator strengths are now 0.015 dex for Fe I $6271 \AA$ and -0.006 dex for Fe I $6609 \AA$. The continuum corrections determined from the inversion are small ( $\sim 1.3-1.6 \%$ of the real continuum intensity). The inferred central wavelengths are almost independent of the continuum correction. The quality of the fit is measured in terms of an equivalent signal-to-noise ratio (SR in the figure), defined as the inverse of the root mean square difference between synthetic and observed profiles.

because of the very broad absorption bands they produce. Strongly blended lines cannot be analyzed unless the blends are taken into account. Another serious problem is line haze, i.e., absorptions in the continuum due to weak spectral features located near the line of interest. Proper modeling of this absorption is difficult because in most cases the blending lines are unidentified. Line haze has long been recognized as an important source of error in abundance and atomic parameter determinations (see, e.g., Holweger et al. 1995). All atomic parameters inferred from the solar spectrum are affected by such uncertainties to some extent. Our approach to minimize the influence of line haze is to apply a wavelength-independent correction $I_{\mathrm{cc}}$ to the synthetic profile $I_{\mathrm{syn}}$. With this correction, the profile which is actually compared with the observations is $I(\lambda)=I_{\mathrm{syn}}(\lambda)-I_{\mathrm{cc}}$, where $I_{\mathrm{syn}}(\lambda)$ is the intensity profile emerging from the two-component model (not affected by line haze). The continuum correction $I_{\mathrm{cc}}$ is treated as a free parameter in the inversion, so it is determined automatically. In Fig. 4 we plot two examples of visible Fe I lines whose oscillator strengths and central wavelenghts have been derived with and without correction. The synthetic profiles without correction (dashed lines) have their continua slightly above the observed ones (dots). In order to compensate for the misfit, at least partially, the synthetic core intensities are smaller than the observed ones. This results in poorer oscillator strengths. The line haze in the examples of Fig. 4 is relatively small, so the atomic parameters derived with and without correction are quite similar. However, the continuum correction may be essential in cases where strong absorption occurs in the continuum due to, e.g., molecular blends or the line being placed in the far wing of a saturated spectral feature.

\subsection{Abundances}

The strength $S$ of a line in the solar spectrum is determined by the product $A g f$, where $A$ stands for the elemental abundance, $g$ is the multiplicity of the lower level, and $f$ is the transition probability. Fitting the observed spectral line yields $S$. Thus, fixing an abundance defines the absolute scale of the oscillator strengths. For iron, Bellot Rubio \& Borrero (2002) have demonstrated that an abundance of 7.43 dex is required for the two-component model to deliver oscillator strengths in the absolute scale of Oxford and Hannover laboratory measurements. This value seems to be appropriate for LTE analyses like ours. For other elements no such determinations have been carried out, so we use the most recent abundance values published in the literature (see Table 1). It is important to stress that changing the abundance is equivalent to changing the oscillator strengths, so rescaling should be no problem if other abundances are employed.

\section{Results}

\subsection{Selection of lines and atomic data}

A list of unblended lines in the solar spectrum from 0.98 to $1.57 \mu \mathrm{m}$ has been compiled using the spectral atlases of Livingston \& Wallace (1991) and Wallace et al. (1998). These 
Table 1. Abundances defining the absolute scale of the oscillator strengths derived in this work. They have been taken from: a Bellot Rubio \& Borrero (2002); b - Asplund et al. (2000c); c - Allende Prieto et al. (2002); and d - Grevesse \& Sauval (1998). These values do not necessarily describe the real Sun.

\begin{tabular}{lcc}
\hline \hline Element & Abundance & Reference \\
\hline $\mathrm{Fe}$ & 7.43 & $\mathrm{a}$ \\
$\mathrm{Si}$ & 7.46 & $\mathrm{~b}$ \\
$\mathrm{C}$ & 8.39 & $\mathrm{c}$ \\
$\mathrm{Cr}$ & 5.67 & $\mathrm{~d}$ \\
$\mathrm{Ca}$ & 6.36 & $\mathrm{~d}$ \\
$\mathrm{Ti}$ & 5.02 & $\mathrm{~d}$ \\
\hline
\end{tabular}

atlases provide line identifications based on line lists dating back to the 1950s and 1960s. Such line lists have been superseded by more recent compilations, e.g., the Vienna Atomic Line Database (VALD; Kupka et al. 1999). Thus, we have confirmed the identification of each line by means of the VALD database. Lines appearing in the atlases but not in VALD were automatically rejected. In addition, the influence of possible blends was assessed for each individual line by considering the transitions of all atomic and ionized species in a wavelength interval of $\pm 2 \AA$ around the line of interest. The relative strengths of the blending lines were estimated by taking into account the elemental abundances, the oscillator strengths given by VALD, and the excitation potentials of the lower levels. Lines considered to be severely affected by blends were removed from the initial list.

Our final list contains 83 lines of the highest quality belonging to 6 different atomic species: $\mathrm{Cr}, \mathrm{Ti}, \mathrm{C}, \mathrm{Si}, \mathrm{Ca}$, and $\mathrm{Fe}$. To the best of our knowledge, these lines are relatively free from blends. Hence, they may be of interest for astrophysical applications. Several lines, including Si I $10827 \AA$ A and Fe I $15648 \AA$, are well known to solar physicists because of their diagnostic capabilities.

\subsection{Observations}

The intensity profiles of the lines selected for analysis have been extracted from the spectral atlas of Brault \& Neckel (1987). This atlas gives the spatially and temporally averaged intensity spectrum of the quiet Sun at disk center. The data were recorded with the Fourier Transform Spectrometer attached to the McMath-Pierce telescope on Kitt Peak. The signal-tonoise ratio of the observations is better than 5000 , with a resolving power $\lambda / \Delta \lambda \simeq 300000$. The atlas is available via ftp at http://www.nso.noao.edu/diglib/ftp.html. The observations are split in several spectral windows. The absolute wavelength calibration of the atlas has been proved to be free from systematic errors between 3290 and $12510 \AA$ (Allende Prieto \& García López 1997). Unfortunately, this has not been tested for lines beyond $1.25 \mu \mathrm{m}$. Thus, in this range we restrict ourselves to determining oscillator strengths only, as we cannot ensure accurate values for central wavelengths.

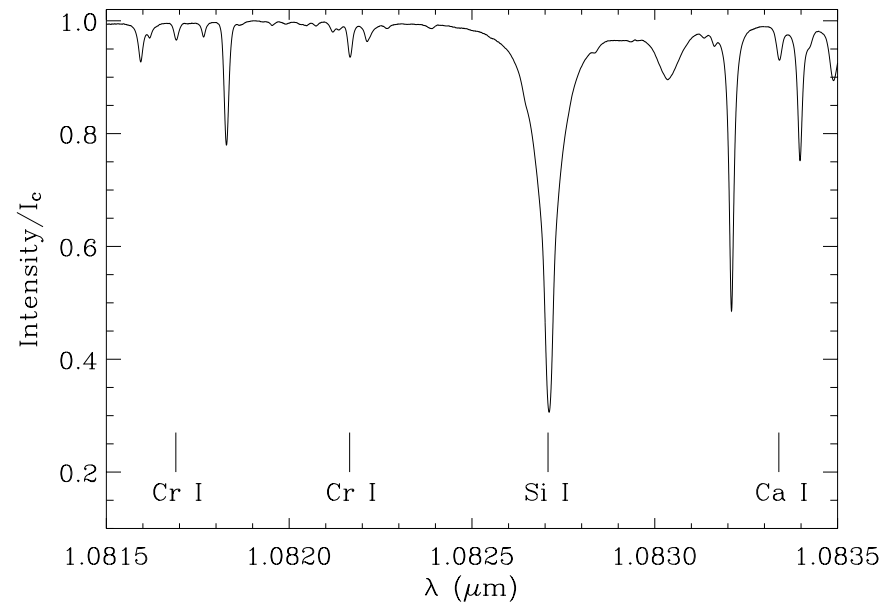

Fig. 5. Section of the FTS atlas of Brault \& Neckel (1987). The lines marked with the element are analyzed in this work. $I_{\mathrm{c}}$ stands for continuum intensity.

Figure 5 shows a small section of the FTS atlas containing four lines analyzed in this work.

The profiles of the 83 lines have been extracted from the atlas by removing the sections of the profiles affected by blends. In order to avoid strong NLTE effects, lines with core intensities below 0.4 (in units of the continuum intensity) have been cut at that level, so only the wings and/or outer core are fitted. Finally, the real continuum (without line haze) has been determined for each line by looking for the maximum continuum intensity in a window of $\pm 4 \AA$ centered at the wavelength of interest.

\subsection{Inferred atomic parameters}

Examples of typical fits obtained from the inversion are shown in Fig. 6 for weak, intermediate, and strong lines. As can be seen, the observed lines are reproduced to a high degree of accuracy. Of course, the fits are much better than those provided by one-component model atmospheres. For most of the lines, the maximum differences between observed and synthetic profiles are smaller than $1 \%$ of the continuum intensity. For strong silicon lines, a larger discrepancy of about $2 \%$ is found in the line core. Apart from that, the fits are very satisfactory. The equivalent width of the lines is reproduced almost perfectly, and the asymmetrical shape of the profiles (observed normally as a stronger and more extended red wing) is also fitted nicely (see, for example, the upper left panel of Fig. 6). The dashed lines in Fig. 6 represent the intensity profiles resulting from Kurucz's oscillator strengths and central wavelengths. It is obvious that neither the equivalent widths nor the central positions of the lines are reproduced with these values. In the four cases of Fig. 6, the corrections to Kurucz's oscillator strengths are on the order of $0.15-0.30$ dex. Figure 6 also illustrates the subtleties of correcting central wavelengths. Naively, one would expect good wavelength corrections by forcing the cores of synthetic and observed lines to match. If this is done for the Ti I line considered in the upper left panel - just by comparing the observed spectrum (dots) with the profile synthesized 

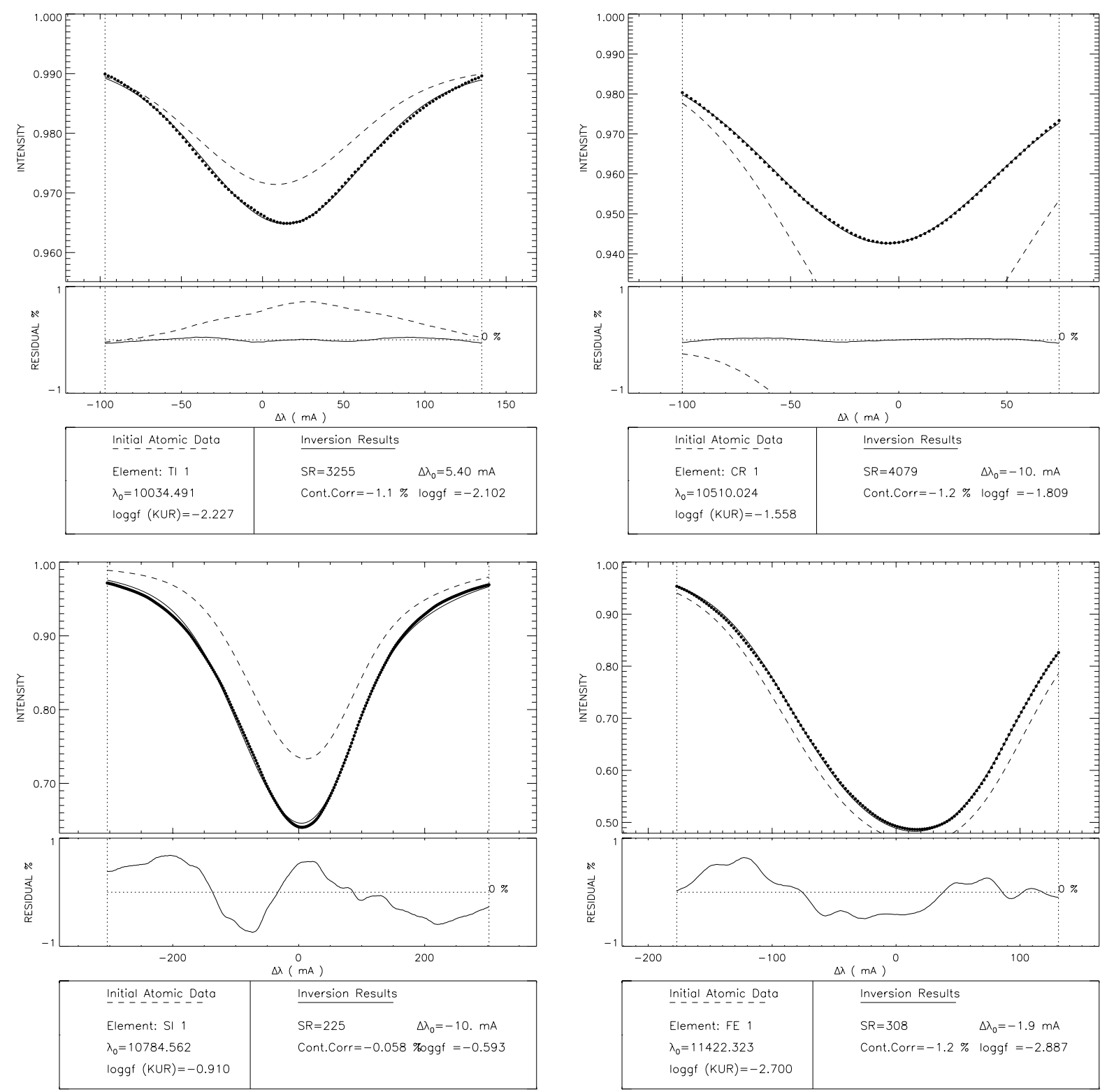

Fig. 6. Typical examples of fits to very weak (upper left and right panels), intermediate (bottom left panel) and strong lines (bottom right panel) with the atomic parameters determined from the inversion. The observed FTS intensity profiles are represented by the dots, whereas the solid lines give the best-fit synthetic profiles. The asymmetric shape of the spectral lines is almost perfectly reproduced, with residuals much smaller than $1 \%$. Dashed lines are synthetic profiles obtained by using the two-component model with Kurucz's oscillator strengths and central wavelengths. Obviously, there is plenty of room for improvement: the strengths of the synthetic and observed lines differ significantly, suggesting wrong $g f$-values. In addition, it is clear that the central positions of the lines are not accurately reproduced using Kurucz's wavelengths.

with Kurucz's atomic parameters (dashed line) - one would find a correction of some $10 \mathrm{~m} \AA$, whereas the correction obtained from the inversion is $5 \mathrm{~m} \AA$. The actual correction is smaller because not only the wavelengh, but also the oscillator strength, is changed in the process, and therefore the line core is formed in a different photospheric layer, where the velocity field is different. This example illustrates the need of simultaneous determinations of oscillator strengths and central wavelengths. Wavelengths obtained from direct comparisons of synthetic and observed profiles may be in error by several $\mathrm{m} \AA$.

The atomic parameters determined from the inversion of the 83 infrared lines, together with some line parameters from the solar spectrum, are given in the Appendix (Tables A.1 and A.2). The fits to the observed profiles are very satisfactory, with typical equivalent signal-to-noise ratios of about 1000 . We note that the typical continuum corrections are smaller than $1 \%$, which lends support to our claim that the lines are relatively free from blends. The uncertainties of our oscillator strengths and central wavelengths are estimated to be $\sim 0.06 \mathrm{dex}$ and $\sim 5 \mathrm{m \AA}$, respectively (see Sect. 5). Thus, we are able to provide atomic parameters with an accuracy similar to that of laboratory measurements.

Figure 7 compares the inferred oscillator strengths and central wavelengths with the semiempirical values of Kurucz (1993, 1994). The rms differences between the solar and Kurucz atomic parameters amount to 0.24 dex and $15.3 \mathrm{~m} \AA$, 
Table 2. Mean and rms differences between our atomic parameters and those by Kurucz $(1993,1994)$ for the various species. Values in parentheses are uncertain because of the small number of lines on which they are based.

\begin{tabular}{lrrrrr}
\hline \hline Species & Lines & $\begin{array}{r}\Delta_{\log g f} \\
(\operatorname{dex})\end{array}$ & $\begin{array}{r}\sigma_{\log g f} \\
(\operatorname{dex})\end{array}$ & $\begin{array}{r}\Delta_{\lambda_{0}} \\
(\mathrm{~m} \AA)\end{array}$ & $\begin{array}{r}\sigma_{\lambda_{0}} \\
(\mathrm{~m} \AA)\end{array}$ \\
\hline Ti I & 6 & -0.16 & 0.14 & 5.5 & 2.3 \\
$\mathrm{Cr}$ I & 7 & -0.15 & 0.06 & 13.9 & 41.7 \\
$\mathrm{Ca}$ I & 4 & -0.02 & $(0.06)$ & 14.0 & $(11.3)$ \\
$\mathrm{Si}$ I & 17 & 0.16 & 0.26 & -1.5 & 10.5 \\
$\mathrm{C} \mathrm{I}$ & 7 & 0.18 & 0.12 & -0.5 & 19.2 \\
Fe I & 42 & 0.05 & 0.32 & 1.8 & 15.7 \\
\hline
\end{tabular}

respectively. The very large scatter is not surprising in view of the relatively poor accuracy of semiempirical calculations. Table 2 gives a more detailed summary of this comparison for the various atomic species.

\section{Accuracy of the inferred atomic parameters}

The best way to estimate the accuracy of the atomic parameters given in Tables A.1 and A.2 is to make comparisons with laboratory measurements. Unfortunately, this is difficult because of the almost complete lack of experimental measurements in the near infrared. Only the central wavelengths of the Fe I lines considered in this work can be compared with the laboratory determinations of Nave et al. (1994). We deem it important to estimate also the accuracy of our oscillator strengths. Therefore, we have decided to apply our procedure to a set of visible Fe I lines of the solar spectrum for which accurate laboratory measurements from Oxford and Hannover exist. The comparison of transition probabilities for visible lines will give us an idea of the accuracy of our determinations in the near infrared.

Figure 8 shows the differences between the central wavelengths of 38 infrared Fe I lines of Table A.2 and the laboratory measurements of Nave et al. (1994). The rms difference is $7.3 \mathrm{m \AA}$. Nave et al. (1994) quote an uncertainty of about $5 \mathrm{~m} \AA$ at $1 \mu \mathrm{m}$. From these values it follows that the uncertainty in our determinations is also $5 \mathrm{m \AA}$ at $1 \mu \mathrm{m}$. The excellent agreement between laboratory wavelengths and those inferred from the solar spectrum is due to our using a two-component model of the quiet photosphere, which allows us to remove the convective blueshifts of the solar lines.

Figure 9a compares the oscillator strengths resulting from the inversion of $60 \mathrm{Fe} I$ lines in the visible part of the solar spectrum with the values provided by Oxford and Hannover. Again, the agreement is quite satisfactory, with an rms difference of only $0.065 \mathrm{dex}$. Since the minimum uncertainty in the laboratory measurements is $0.03 \mathrm{dex}$, we conclude that our oscillator strengths are uncertain by less than $0.057 \mathrm{dex}$. This is indeed an excellent result. We not only improve on earlier solar determinations based on one-component model atmospheres, but also some laboratory measurements (compare Figs. 1 and 2 with Fig. 9a). At this point it is necessary to mention that Thévenin $(1989,1990)$ and Gurtovenko \& $\operatorname{Kostik}(1981,1982)$ quote uncertainties of only 0.05 dex in their solar oscillator strengths.
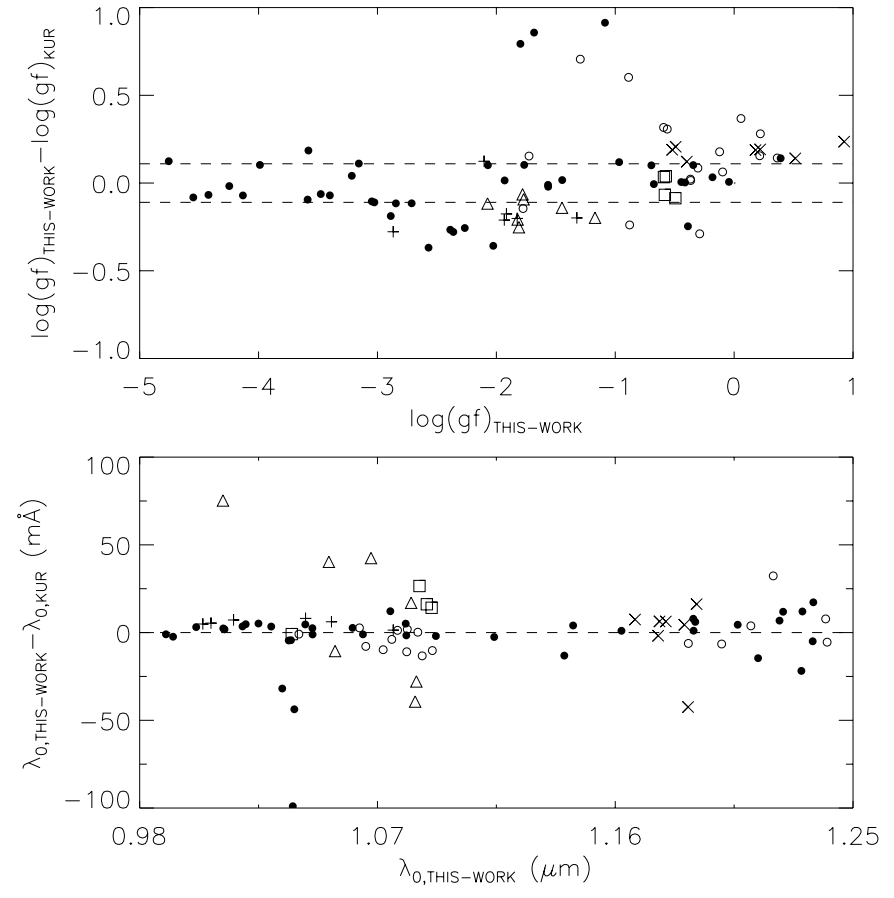

Fig. 7. Comparison between the atomic parameters derived in this work and the semiempirical calculations of $\operatorname{Kurucz}(1993,1994)$. Top: oscillator strengths. The horizontal dashed lines indicate differences of \pm 0.1 dex. Bottom: Central wavelengths. Symbols are as follows: $\mathrm{Ti}(+), \mathrm{Cr}(\triangle), \mathrm{Ca}(\square), \mathrm{C}(\times), \mathrm{Si}(\circ)$, and $\mathrm{Fe}(\bullet)$.

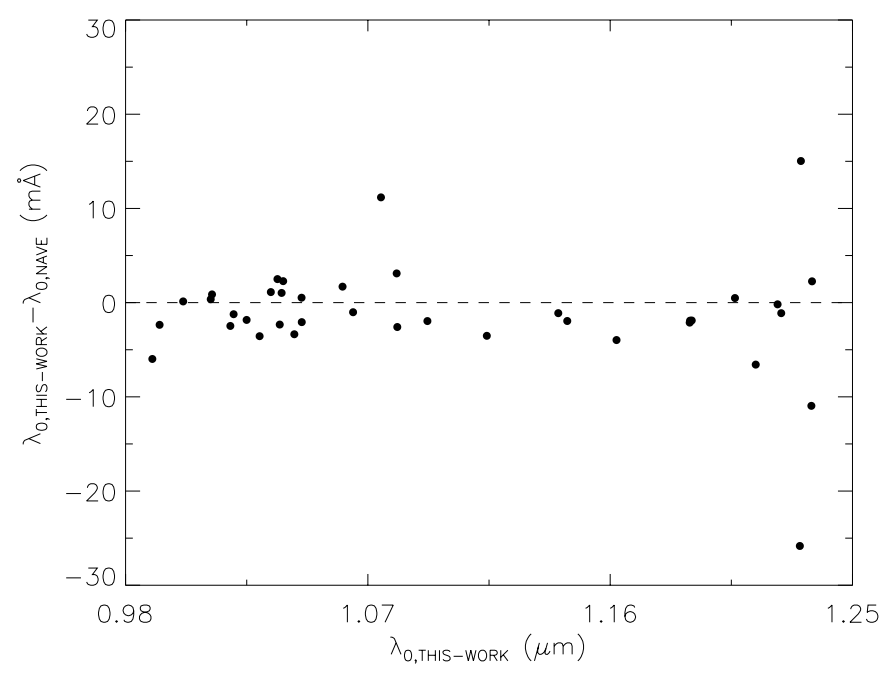

Fig. 8. Comparison of central wavelengths determined from the solar spectrum and laboratory wavelengths of Nave et al. (1994) for the $38 \mathrm{Fe}$ I lines of Table A.2 in the interval $0.98-1.25 \mu \mathrm{m}$. The mean and rms differences are $-0.2 \mathrm{~m} \AA$ and $7.3 \mathrm{~m} \AA$, respectively.

From Fig. 2 we believe that their real uncertainty is much larger, probably 0.14 dex or more.

Figure 9a demonstrates that the differences between our $g f$-values and those measured in the laboratory do not depend on line strength. Any dependence would reveal systematic errors in the solar determination. The lack of trends in Fig. 9a is mainly the result of a realistic estimation of the collisional broadening based on the quantum mechanical formulation of $\mathrm{ABO}$. This is shown in Figs. 9b and 9d, where the 

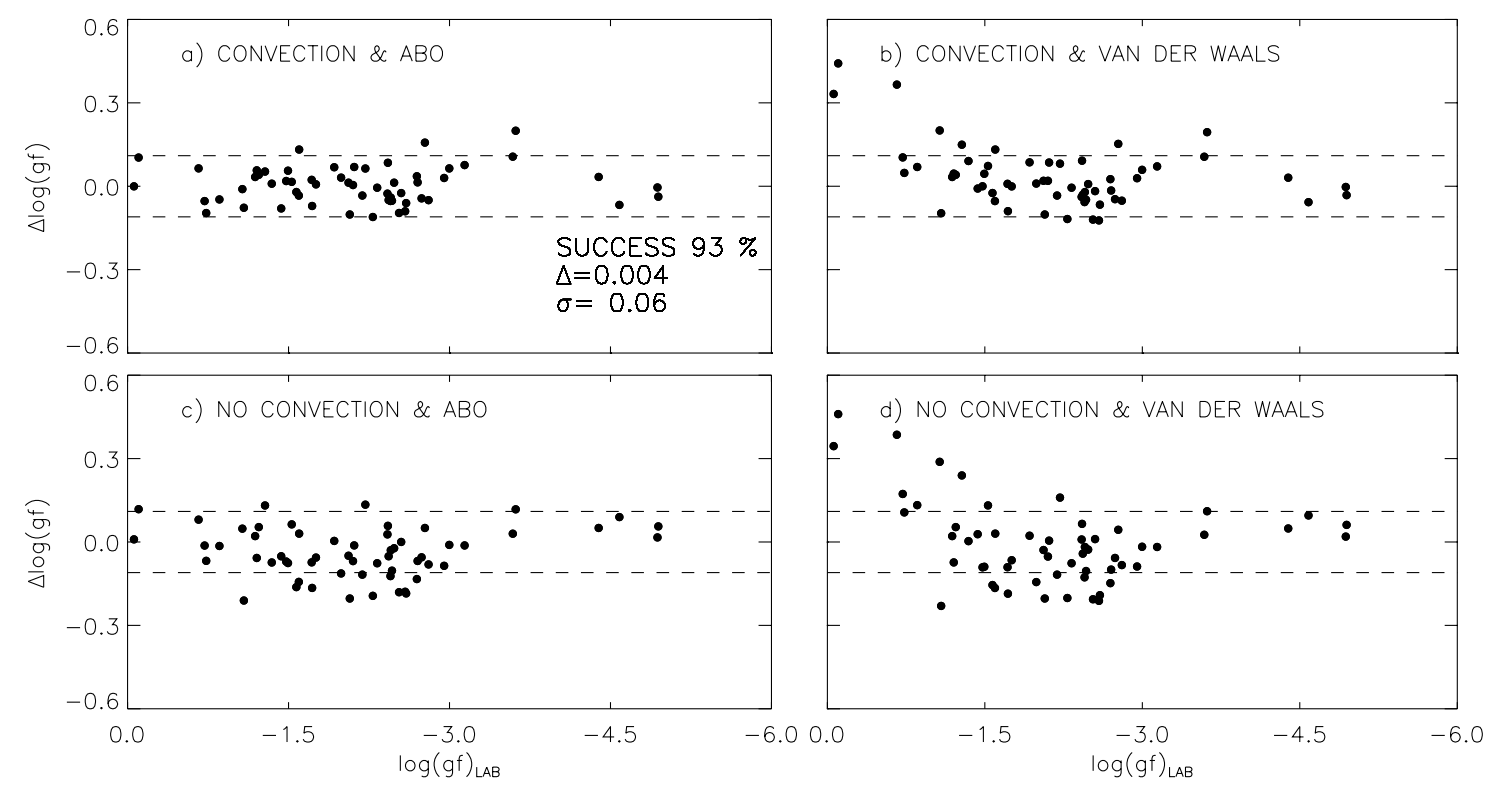

Fig. 9. a) Comparison between the oscillator strengths of 60 visible Fe I lines determined from the solar spectrum and from laboratory measurements. The rms difference turns out to be 0.065 dex. The horizontal lines indicate differences of \pm 0.1 dex. The effects of using a simplified procedure for estimating $g f$-values from the solar spectrum are displayed in the other panels. b) Solar determinations based on van der Waals broadening instead of quantum mechanical parameters from ABO. c) Solar determinations based on quantum mechanical broadening parameters but no account of convective motions (the model velocities are artificially set to zero). d) Solar oscillator strengths based on van der Waals broadening and no account of convective velocities. Note the similarity between this panel and Fig. 2.

same comparison of oscillator strengths is repeated, now with the solar $g f$-values resulting from van der Waals broadening ${ }^{2}$. A clear trend with $\log g f$ is apparent. The use of a realistic two-component model of the solar photosphere also improves the quality of the oscillator strengths, as a cursory glance at Figs. 9a and 9c demonstrates. To produce Fig. 9c, the collisional broadening was computed according to the ABO theory, but the velocities of the two-component model were set to zero.

To summarize, our method increases the accuracy of the atomic parameters determined in previous analyses of the solar spectrum thanks to 1) an improved treatment of the broadening of spectral lines by collisions with neutral hydrogen, and 2) the realistic modeling of convective motions in the solar photosphere provided by the two-component model of BBR.

\section{Conclusions}

We have presented a method for determining very accurate oscillator strengths and central wavelengths from the solar spectrum. The method is based on the fitting of the intensity profiles of lines emerging from the quiet Sun. Comparisons between the inferred atomic parameters and precise laboratory measurements show that the oscillator strengths and central wavelengths determined in this way are accurate to about $0.06 \mathrm{dex}$ and $5 \mathrm{m \AA}$ at $1 \mu \mathrm{m}$, respectively. Our results have an accuracy comparable with that of the best laboratory measurements. This is due to the realistic description of the solar photosphere provided by the two-component model of Borrero \& Bellot Rubio (2002) and the use of the collisional broadening theory developed by Anstee, Barklem, \& O’Mara.

${ }^{2}$ The classical Unsöld formula is adopted together with an enhancement factor $E=2.0$, which is in between the values used by Gurtovenko \& Kostik $(E=1.5)$ and Thévenin $(E=2.5)$.
The small uncertainties in our oscillator strengths suggest that errors in the model atmosphere and broadening parameters for the lines used cannot be very large. They also indicate that our assumption of LTE is reasonable. NLTE effects would produce systematic differences between the solar and laboratory transition probabilities. Such differences are not observed.

We have applied the method to 83 near-infrared lines belonging to six different atomic species. Many of these lines are of interest for astrophysical applications, but none has been measured in the laboratory (except the Fe I lines, whose wavelengths were determined by Nave et al. 1994). This demonstrates that the method is a powerful tool for characterizing atomic transitions in the infrared. Of course, it can also be applied to visible lines. The main advantage of using the Sun as a laboratory is that we are not restricted to transitions excitable on Earth. We plan to carry out a systematic study of the solar spectrum in order to determine accurate oscillator strengths and central wavelengths for a large number of spectral lines. The availability of precise atomic parameters will enhance the diagnostic capabilities of high resolution spectroscopic observations. Very accurate wavelengths are also important for revising the energy levels of atoms and ions that have not been studied in the laboratory.

Acknowledgements. This work has been partially funded by the Deutsche Forschungsgemeinschaft, and by the Spanish Ministry of Science and Technology under projects PNAYA2001-1649 and PNAYA2001-1177. PB acknowledges support from the Swedish Research Council. NSO Kitt Peak FTS data used here were produced by NSF/NOAO. This research has made use of NASA's Astrophysics Data System Bibliographic Services. 


\section{Appendix A: Atomic data}

Table A.1. Atomic parameters derived is this work for $\mathrm{Ti}, \mathrm{Cr}, \mathrm{Ca}, \mathrm{Si}$, and $\mathrm{C}$ near-infrared lines. $\lambda_{0}$ represents the central wavelength (already corrected for convective blueshift and gravitational redshift), $\chi_{\ell}$ the excitation potential of the lower level, $\log g f$ the logarithm of the oscillator strength times the multiplicity of the lower level, $\alpha$ and $\sigma$ the collisional broadening parameters ( $a_{0}$ is Bohr radius), $\gamma_{\text {rad }}$ the radiative damping, $I_{\min }$ the line core intensity in the solar spectrum, $W_{\lambda}$ the equivalent width of the line in the solar spectrum, and $I_{\mathrm{cc}}$ the continuum correction discussed in Sect. 3.5. The Landé factors correspond to LS coupling. The oscillator strengths given here should be used together with the abundances of Table 1, or rescaled otherwise. Best fits to the solar spectrum are achieved by including the continuum correction $I_{\mathrm{cc}}$.

\begin{tabular}{|c|c|c|c|c|c|c|c|c|c|c|}
\hline Species & $\begin{array}{l}\lambda_{0}^{\mathrm{a}} \\
(\AA) \\
\end{array}$ & $\begin{array}{c}\chi_{\ell}^{\mathrm{b}} \\
(\mathrm{eV})\end{array}$ & $\begin{array}{r}\log g f^{\mathrm{a}} \\
(\mathrm{dex})\end{array}$ & $\alpha^{\mathrm{a}}$ & $\begin{array}{r}\sigma^{\mathrm{a}} \\
\left(a_{0}^{2}\right) \\
\end{array}$ & $\begin{array}{c}\log \gamma_{\mathrm{rad}}^{\mathrm{b}} \\
\left(\mathrm{s}^{-1}\right)\end{array}$ & Landé factor & $I_{\min }$ & $\begin{array}{r}W_{\lambda} \\
(\mathrm{m} \AA) \\
\end{array}$ & $\begin{array}{l}I_{\mathrm{cc}} \\
(\%) \\
\end{array}$ \\
\hline Ti I & 10003.091 & 2.160 & -1.323 & 0.248 & 321 & 6.746 & 1.17 & 0.96 & 4.4 & 0.9 \\
\hline Ti I & 10034.496 & 1.460 & -2.103 & 0.264 & 278 & 6.467 & 1.11 & 0.96 & 3.7 & 1.2 \\
\hline Ti I & 10120.902 & 2.175 & -1.933 & 0.247 & 327 & 6.746 & 1.51 & 0.99 & 1.1 & 0.6 \\
\hline Ti I & 10396.810 & 0.848 & -1.825 & 0.256 & 257 & 5.053 & 1.13 & 0.81 & 25.6 & 1.6 \\
\hline Ti I & 10496.120 & 0.836 & -1.915 & 0.257 & 256 & 5.041 & 1.05 & 0.84 & 22.2 & 0.9 \\
\hline Ti I & 10732.856 & 0.826 & -2.867 & 0.258 & 255 & 5.037 & 1.09 & 0.97 & 3.1 & 0.8 \\
\hline $\mathrm{Cr} \mathrm{I}$ & 10080.366 & 3.556 & -1.448 & 0.243 & 266 & 6.500 & 0.52 & 0.96 & 4.4 & 1.0 \\
\hline $\mathrm{Cr} \mathrm{I}$ & 10486.252 & 3.011 & -1.170 & 0.253 & 275 & 6.773 & 1.50 & 0.83 & 24.7 & 1.5 \\
\hline $\mathrm{CrI}$ & 10510.013 & 3.013 & -1.810 & 0.253 & 275 & 6.772 & 1.42 & 0.94 & 6.7 & 1.2 \\
\hline $\mathrm{CrI}$ & 10647.648 & 3.011 & -1.821 & 0.252 & 274 & 6.768 & 1.53 & 0.94 & 6.7 & 1.2 \\
\hline $\mathrm{Cr} \mathrm{I}$ & 10801.363 & 3.011 & -1.779 & 0.251 & 273 & 6.607 & 1.51 & 0.94 & 7.5 & 0.7 \\
\hline $\mathrm{Cr} \mathrm{I}$ & 10816.909 & 3.013 & -2.074 & 0.251 & 273 & 6.607 & 1.50 & 0.96 & 3.9 & 1.0 \\
\hline $\mathrm{CrI}$ & 10821.662 & 3.013 & -1.771 & 0.251 & 273 & 6.607 & 1.60 & 0.93 & 7.7 & 1.5 \\
\hline $\mathrm{Ca} \mathrm{I}$ & 10343.819 & 2.933 & -0.494 & 0.221 & 1015 & 8.404 & 1.00 & 0.46 & 147.7 & 1.1 \\
\hline $\mathrm{Ca} \mathrm{I}$ & 10833.409 & 4.877 & -0.582 & 0.278 & 586 & 8.330 & 1.50 & 0.93 & 8.8 & 2.3 \\
\hline $\mathrm{Ca} \mathrm{I}$ & 10861.598 & 4.877 & -0.574 & 0.278 & 585 & 8.328 & 1.50 & 0.95 & 8.8 & 0.8 \\
\hline $\mathrm{Ca} \mathrm{I}$ & 10879.882 & 4.877 & -0.590 & 0.278 & 584 & 8.326 & 1.50 & 0.95 & 8.4 & 0.6 \\
\hline $\mathrm{Si} \mathrm{I}$ & 10371.263 & 4.930 & -0.879 & 0.231 & 741 & & 1.75 & 0.46 & 172.0 & 3.0 \\
\hline Si I & 10603.428 & 4.930 & -0.365 & 0.230 & 729 & & 1.50 & 0.40 & 263.1 & 1.5 \\
\hline Si I & 10627.640 & 5.863 & -0.289 & 0.311 & 1263 & & 1.75 & 0.54 & 148.8 & 0.0 \\
\hline Si I & 10694.242 & 5.964 & 0.221 & 0.292 & 1453 & & 1.00 & 0.47 & 230.4 & 1.0 \\
\hline Si I & 10727.402 & 5.984 & 0.216 & 0.288 & 1403 & & 1.12 & 0.44 & 238.1 & 3.9 \\
\hline Si I & 10749.379 & 4.930 & -0.122 & 0.230 & 722 & & 1.50 & 0.37 & 318.2 & 0.0 \\
\hline Si I & 10784.551 & 5.964 & -0.593 & 0.294 & 1424 & & 0.92 & 0.64 & 101.3 & 0.1 \\
\hline Si I & 10786.851 & 4.930 & -0.305 & 0.230 & 721 & & 1.50 & 0.40 & 277.6 & 0.8 \\
\hline Si I & 10827.089 & 4.954 & 0.363 & 0.231 & 729 & & 1.50 & 0.31 & 515.0 & 0.0 \\
\hline Si I & 10843.845 & 5.863 & 0.058 & 0.311 & 1212 & & 1.00 & 0.47 & 210.4 & 0.8 \\
\hline Si I & 10882.799 & 5.984 & -0.562 & 0.291 & 1453 & & 1.21 & 0.64 & 106.6 & 0.0 \\
\hline Si I & 11863.914 & 5.984 & -1.294 & 0.303 & 1244 & & 1.17 & 0.83 & 43.3 & 0.3 \\
\hline Si I & 11991.561 & 4.920 & -0.097 & 0.228 & 675 & & 0.50 & 0.37 & 380.2 & 0.0 \\
\hline Si I & 12103.539 & 4.930 & -0.368 & 0.228 & 675 & & 1.00 & 0.41 & 297.0 & 1.1 \\
\hline Si I & 12189.273 & 6.616 & -0.888 & 0.319 & 3138 & & 1.12 & 0.90 & 32.9 & 0.0 \\
\hline Si I & 12390.162 & 5.082 & -1.725 & 0.236 & 731 & & 1.25 & 0.69 & 84.5 & 0.8 \\
\hline Si I & 12395.827 & 4.854 & -1.775 & 0.225 & 671 & & 2.00 & 0.63 & 102.0 & 1.4 \\
\hline C I & 11659.684 & 8.647 & 0.220 & 0.270 & 764 & & 1.33 & 0.73 & 121.6 & 0.0 \\
\hline C I & 11748.230 & 8.640 & 0.515 & 0.270 & 749 & & 0.75 & 0.67 & 160.3 & 0.0 \\
\hline C I & 11753.320 & 8.647 & 0.926 & 0.270 & 756 & & 1.12 & 0.61 & 209.8 & 0.0 \\
\hline C I & 11777.552 & 8.643 & -0.398 & 0.271 & 749 & & 0.92 & 0.83 & 64.6 & 0.2 \\
\hline C I & 11848.711 & 8.643 & -0.491 & 0.223 & 1384 & & 1.33 & 0.85 & 61.2 & 0.0 \\
\hline C I & 11862.964 & 8.640 & -0.520 & 0.222 & 1377 & & 1.00 & 0.86 & 59.2 & 0.0 \\
\hline C I & 11895.771 & 8.647 & 0.180 & 0.223 & 1385 & & 1.17 & 0.74 & 130.0 & 0.0 \\
\hline
\end{tabular}

a Derived in this work.

b VALD database (reference points to Kurucz 1993, 1994). 
Table A.2. Same as Table A.1, but for neutral iron lines.

\begin{tabular}{|c|c|c|c|c|c|c|c|c|c|c|}
\hline Species & $\begin{array}{r}\lambda_{0}^{\mathrm{a}} \\
(\AA)\end{array}$ & $\begin{array}{c}\chi_{\ell}^{\mathrm{b}} \\
(\mathrm{eV})\end{array}$ & $\begin{array}{r}\log g f^{\mathrm{a}} \\
\quad(\operatorname{dex})\end{array}$ & $\overline{\alpha^{\mathrm{a}}}$ & $\begin{array}{c}\sigma^{\mathrm{a}} \\
\left(a_{0}^{2}\right)\end{array}$ & $\begin{array}{c}\log \gamma_{\text {rad }}^{\mathrm{b}} \\
\left(\mathrm{s}^{-1}\right)\end{array}$ & Landé factor & $\overline{I_{\min }}$ & $\begin{array}{r}W_{\lambda} \\
(\mathrm{m} \AA)\end{array}$ & $\begin{array}{l}I_{\mathrm{cc}} \\
(\%)\end{array}$ \\
\hline $\mathrm{Fe} \mathrm{I}$ & 9861.731 & 5.064 & -0.388 & 0.278 & 767 & 8.812 & 1.21 & 0.58 & 79.3 & 1.8 \\
\hline $\mathrm{Fe} \mathrm{I}$ & 9889.034 & 5.033 & -0.344 & 0.280 & 735 & 8.802 & 1.42 & 0.57 & 84.6 & 0.5 \\
\hline $\mathrm{Fe} \mathrm{I}$ & 9977.641 & 5.064 & -1.683 & 0.278 & 752 & 8.874 & 1.46 & 0.93 & 9.4 & 0.9 \\
\hline Fe I & 10081.394 & 2.424 & -4.421 & 0.250 & 208 & 7.292 & 2.00 & 0.95 & 6.5 & 0.7 \\
\hline $\mathrm{Fe} \mathrm{I}$ & 10086.242 & 2.949 & -3.989 & 0.265 & 272 & 8.079 & 1.31 & 0.95 & 5.4 & 1.1 \\
\hline $\mathrm{Fe} \mathrm{I}$ & 10155.163 & 2.176 & -4.246 & 0.256 & 212 & 7.176 & 1.46 & 0.88 & 16.0 & 0.7 \\
\hline $\mathrm{Fe} I$ & 10167.468 & 2.198 & -4.132 & 0.256 & 212 & 7.164 & 1.41 & 0.85 & 19.3 & 0.9 \\
\hline $\mathrm{Fe} \mathrm{I}$ & 10216.312 & 4.733 & -0.182 & 0.224 & 893 & 8.281 & 1.23 & 0.48 & 138.2 & 1.0 \\
\hline $\mathrm{Fe} \mathrm{I}$ & 10265.216 & 2.223 & -4.549 & 0.256 & 213 & 7.155 & 1.24 & 0.94 & 7.8 & 0.6 \\
\hline $\mathrm{Fe} \mathrm{I}$ & 10307.455 & 4.593 & -2.387 & 0.249 & 236 & 8.064 & 1.03 & 0.96 & 5.7 & 0.6 \\
\hline $\mathrm{Fe} \mathrm{I}$ & 10332.331 & 3.635 & -3.048 & 0.304 & 358 & 8.161 & 0.50 & 0.92 & 10.0 & 0.7 \\
\hline $\mathrm{Fe} \mathrm{I}$ & 10340.884 & 2.198 & -3.587 & 0.256 & 212 & 7.176 & 0.68 & 0.67 & 49.0 & 0.3 \\
\hline $\mathrm{Fe} \mathrm{I}$ & 10347.967 & 5.393 & -0.696 & 0.282 & 844 & 8.475 & 1.34 & 0.79 & 36.8 & 1.1 \\
\hline $\mathrm{Fe} \mathrm{I}$ & 10353.809 & 5.393 & -0.967 & 0.282 & 843 & 8.477 & 1.46 & 0.86 & 23.2 & 1.2 \\
\hline $\mathrm{Fe} \mathrm{I}$ & 10395.794 & 2.176 & -3.400 & 0.256 & 211 & 7.193 & 0.89 & 0.60 & 64.0 & 0.0 \\
\hline $\mathrm{Fe} \mathrm{I}$ & 10423.031 & 2.692 & -3.581 & 0.253 & 227 & 6.328 & 1.09 & 0.84 & 22.3 & 0.7 \\
\hline $\mathrm{Fe} \mathrm{I}$ & 10423.743 & 3.071 & -3.026 & 0.248 & 242 & 6.886 & 1.48 & 0.79 & 30.6 & 0.3 \\
\hline $\mathrm{Fe} \mathrm{I}$ & 10577.143 & 3.301 & -3.157 & 0.272 & 305 & 7.879 & 0.84 & 0.88 & 16.2 & 0.7 \\
\hline $\mathrm{Fe} \mathrm{I}$ & 10616.722 & 3.267 & -3.216 & 0.273 & 300 & 7.905 & 0.90 & 0.89 & 15.5 & 0.4 \\
\hline $\mathrm{Fe} \mathrm{I}$ & 10721.668 & 5.507 & -1.766 & 0.271 & 917 & 8.137 & 0.94 & 0.97 & 3.6 & 0.8 \\
\hline $\mathrm{Fe} I$ & 10780.697 & 3.237 & -3.477 & 0.274 & 296 & 7.940 & 0.96 & 0.93 & 9.8 & 0.5 \\
\hline $\mathrm{Fe} \mathrm{I}$ & 10783.048 & 3.111 & -2.712 & 0.248 & 243 & 6.886 & 1.50 & 0.69 & 49.1 & 0.3 \\
\hline $\mathrm{Fe} I$ & 10896.300 & 3.071 & -2.845 & 0.249 & 240 & 7.196 & 1.51 & 0.72 & 43.6 & 0.4 \\
\hline $\mathrm{Fe} \mathrm{I}$ & 11119.794 & 2.845 & -2.570 & 0.256 & 239 & 6.820 & 1.00 & 0.53 & 84.6 & 3.7 \\
\hline $\mathrm{Fe} \mathrm{I}$ & 11388.539 & 5.620 & -0.806 & 0.226 & 838 & & 1.67 & 0.85 & 24.6 & 1.9 \\
\hline $\mathrm{Fe} \mathrm{I}$ & 11422.321 & 2.198 & -2.888 & 0.258 & 210 & 7.146 & 1.98 & 0.49 & 110.5 & 1.2 \\
\hline $\mathrm{Fe} I$ & 11607.571 & 2.198 & -2.265 & 0.258 & 210 & 7.152 & 1.66 & 0.43 & 163.4 & 0.6 \\
\hline $\mathrm{Fe} \mathrm{I}$ & 11882.845 & 2.198 & -2.026 & 0.258 & 210 & 7.161 & 1.18 & 0.41 & 197.1 & 2.5 \\
\hline $\mathrm{Fe} \mathrm{I}$ & 11884.083 & 2.223 & -2.362 & 0.258 & 210 & 7.152 & 1.00 & 0.44 & 158.5 & 1.1 \\
\hline $\mathrm{Fe} \mathrm{I}$ & 11890.488 & 5.539 & -0.413 & 0.224 & 1080 & 8.029 & 1.12 & 0.71 & 61.9 & 3.6 \\
\hline $\mathrm{Fe} \mathrm{I}$ & 12053.083 & 4.558 & -1.564 & 0.245 & 821 & 8.363 & 1.58 & 0.79 & 41.7 & 0.7 \\
\hline $\mathrm{Fe} \mathrm{I}$ & 12131.168 & 5.947 & -1.086 & 0.311 & 1839 & & 1.25 & 0.96 & 7.9 & 0.2 \\
\hline $\mathrm{Fe} I$ & 12213.333 & 4.638 & -1.930 & 0.238 & 851 & 8.336 & 2.50 & 0.89 & 19.1 & 1.4 \\
\hline $\mathrm{Fe} \mathrm{I}$ & 12227.112 & 4.607 & -1.446 & 0.242 & 835 & 8.346 & 1.67 & 0.76 & 48.6 & 1.6 \\
\hline $\mathrm{Fe} \mathrm{I}$ & 12297.131 & 4.913 & -1.798 & 0.243 & 882 & 8.656 & 1.87 & 0.90 & 15.1 & 2.9 \\
\hline $\mathrm{Fe} \mathrm{I}$ & 12301.082 & 5.446 & -2.071 & 0.232 & 771 & 8.333 & 1.39 & 0.98 & 2.7 & 1.1 \\
\hline $\mathrm{Fe} \mathrm{I}$ & 12340.476 & 2.279 & -4.755 & 0.252 & 197 & 7.146 & 1.51 & 0.95 & 6.2 & 1.3 \\
\hline $\mathrm{Fe} \mathrm{I}$ & 12342.916 & 4.638 & -1.565 & 0.239 & 845 & 8.336 & 2.01 & 0.80 & 38.8 & 1.7 \\
\hline $\mathrm{Fe} \mathrm{I}$ & $15588.264^{*}$ & 6.474 & 0.391 & 0.227 & 952 & & 1.50 & 0.69 & 111.0 & 1.7 \\
\hline $\mathrm{Fe} \mathrm{I}$ & $15590.051^{*}$ & 6.241 & -0.444 & 0.330 & 1441 & & 1.47 & 0.86 & 39.9 & 1.9 \\
\hline $\mathrm{Fe} \mathrm{I}$ & $15648.515^{*}$ & 5.426 & -0.675 & 0.229 & 977 & & 2.98 & 0.70 & 94.9 & 1.6 \\
\hline $\mathrm{Fe} \mathrm{I}$ & $15652.874^{*}$ & 6.246 & -0.043 & 0.330 & 1445 & & 1.50 & 0.77 & 83.2 & 0.8 \\
\hline
\end{tabular}

a Derived in this work.

b VALD database (reference points to Kurucz 1993, 1994).

* Central wavelengths taken from Nave et al. (1994).

\section{References}

Allende Prieto, C., \& García López, R. 1997, A\&AS, 129, 41 Allende Prieto, C., Lambert, D. L., \& Asplund, M. 2002, ApJ, 573, L137

Anstee, S. D., \& O’Mara, B. J. 1991, MNRAS, 253, 549 Anstee, S. D., \& O'Mara, B. J. 1995, MNRAS, 276, 859
Asplund, M., Ludwig, H. G., Nordlund, A., \& Stein, R. F. 2000a, A\&A, 359, 669

Asplund, M., Nordlund, Å., Trampedach, R., Allende Prieto, C., \& Stein, R. F. 2000b, A\&A, 359, 729

Asplund, M., Nordlund, Å., Trampedach, R., \& Stein, R. F. 2000c, A\&A, 359, 743 
Bard, A., Kock, A., \& Kock, M. 1991, A\&A, 248, 315

Bard, A., \& Kock, M. 1994, A\&A, 282, 1014

Barklem, P. S., \& O'Mara, B. J. 1997, MNRAS, 290, 102 (ABO)

Barklem, P. S., \& O'Mara, B. J. 2001, J. Phys. B., 34, 4785

Barklem, P. S., O’Mara, B. J., \& Ross, J. E. 1998, MNRAS, 296, 1057 (ABO)

Barklem, P. S., Piskunov, N., \& O’Mara, B. J. 2000, A\&AS, 142, 467

Bellot Rubio, L. R., \& Borrero, J. M. 2002, A\&A, 391, 331

Bellot Rubio, L. R., Ruiz Cobo, B., \& Collados, M. 1998, ApJ, 506, 805

Bellot Rubio, L. R., Ruiz Cobo, B., \& Collados, M. 1999, A\&A, 341, L31

Blackwell, D. E. 1990, in Proc. 3rd International Colloq. of the Royal Netherlands Academy of Arts and Sciences, ed. J. E. Hansen (Amsterdam: North-Holland), 160

Blackwell, D. E., \& Collins, B. S. 1972, MNRAS, 157, 255

Blackwell, D. E., Ibbetson, P. A., Petford, A. D., \& Willis, R. B. 1976, MNRAS, 177, 219

Blackwell, D. E., Petford, A. D., \& Shallis, M. J. 1979, MNRAS, 186, 657

Blackwell, D. E., Petford, A. D., \& Simmons, G. J. 1982, MNRAS, 201, 595

Blackwell, D. E., Booth, A. J., Haddock, D. J., Petford, A. D., \& Leggett, S. K. 1986, MNRAS, 220, 549

Borrero, J. M., \& Bellot Rubio, L. R. 2002, A\&A, 385, 1056 (BBR)

Brault, J., \& Neckel, H. 1987, Spectral Atlas of Solar Absolute Disk-Averaged and Disk-Center Intensity from 3290 to $12510 \AA$, unpublished

Frutiger, C., Solanki, S. K., Fligge, M., \& Bruls, J. H. M. J. 2000, A\&A, 358, 1109

Gingerich, O., Noyes, R. W., \& Kalkofen, W. 1971, Sol. Phys., 18, 347

Grevesse, N., \& Sauval, A. J. 1998, Space Sci. Rev., 85, 161

Gurtovenko, E. A., \& Kostik, R. I. 1981, A\&AS, 46, 239

Gurtovenko, E. A., \& Kostik, R. I. 1982, A\&AS, 47, 193

Gustafsson, B., Bell, R. A., Ericksson, K., \& Nordlund, A.. 1975, A\&A, 57, 235

Holweger, H., \& Müller, E. A. 1974, Sol. Phys., 39, 19

Holweger, H., Kock, M., \& Bard, A. 1995, A\&A, 296, 233

Johansson, S. 1978, Phys. Scr., 18, 217

Johansson, S. 1995, ASP Conf. Ser., 81, 151

Johansson, S. 2002, Highlights Astron., 12, 84

Kupka, F., Piskunov, N., Ryabchikova, T. A., Stempels, H. C., \& Weiss, W. W. 1999, A\&AS, 138, 119
Kurucz, R. L. 1993, SAO, CDROM 18

Kurucz, R. L. 1994, SAO, CDROM 20-22

Kurucz, R. L. 1995, ASP Conf. Ser., 78, 205

Kurucz, R. L. 2003, in IAU Symp. 210, Modelling of Stellar Atmospheres, ed. N. E. Piskunov, W. W. Weiss, \& D. F. Gray, in press

Kurucz, R. L., \& Peytremann, E. 1975, SAO Special Rep. 362

Learner, R. C. M., \& Thorne, A. P. 1988, JOSA B, 5, 2045

Litzén, U. 1995, ASP Conf. Ser., 81, 167

Livingston, W., \& Wallace, L. 1991, An Atlas of the Solar Spectrum in the infrared from 1.1 to 5.4 microns, NSO Technical Rep. 91-101

Martínez Pillet, V., Collados, M., Sánchez Almeida, J., et al. 1999, ASP Conf. Ser., 183, 264

May, M., Richter, J., \& Wichelmann, J. 1974, A\&AS, 18, 405

Nave, G., Johansson, S., Learner, R. C. M., Thorne, A. P., \& Brault, J. W. 1994, ApJS, 94, 221

O'Brian, T. R., Wickliffe, M. E., Lawler, J. E., Whaling, J. W., \& Brault, W. 1991, JOSA B, 8, 1185

Pickering, J. C., Thorne, A. P., \& Blackwell-Whitehead, R. 2002, in NASA Laboratory Astrophysics Workshop, Abstract Book, ed. Farid Salama, 72

Pickering, J. C., Raassen, A. J. J., Uylings, P. H. M., \& Johansson, S. 1998, ApJS, 117, 261

Ruiz Cobo, B., \& del Toro Iniesta, J. C. 1992, ApJ, 398, 375

Ruiz Cobo, B., \& del Toro Iniesta, J. C. 1994, A\&A, 283, 129

Seaton, M. J., Yan, Y., Mihalas, D., \& Pradhan, A. K. 1994, MNRAS, 266, 805

Steffen, M., \& Holweger, H. 2002, A\&A, 387, 258

Stein, R. F., \& Nordlund, Å. 1998, ApJ, 499, 914

Thévenin, F. 1989, A\&AS, 77, 137

Thévenin, F. 1990, A\&AS, 82, 179

del Toro Iniesta, J. C. 2003, Introduction to Spectropolarimetry (Cambridge: Cambridge University Press)

Unsöld, A. 1955, Physik der Sternatmosphären (Berlin: Springer)

Uylings, P. H. M., \& Raassen, A. J. J. 1997, A\&AS, 125, 539

Wallace, L., Livingston, W., Bernath, P. F., \& Ram, R. S. 1998, An Atlas of the Sunspot Umbral Spectrum in the Red and Infrared from 6642 to $11230 \AA$, NSO Technical Rep. 1998-002

Wiedemann, G., Delabre, B., Huster, G., Moorwood, A. F., \& Sokar, B. 2000, Proc. SPIE, 4008, 1076

Wittmann, A. 1974, Sol. Phys., 35, 11 